\title{
Digital superresolution in seismic AVO inversion
}

\author{
Odd Kolbjørnsen ${ }^{1,2}$, Andreas Kjelsrud Evensen ${ }^{1}$, Espen Harris Nilsen ${ }^{1}$, and Jan-Erik Lie ${ }^{1}$ \\ https://doi.org/10.1190/tle38100791.1
}

\begin{abstract}
The sparseness promoted by the total variation norm is utilized to achieve superresolution amplitude-variation-with-offset (AVO) inversion. The total variation norm promotes solutions that have constant values within unspecified regions and thus are well suited for an earth model consisting of layers bounded by faults and erosion surfaces. Algorithmic developments from digital image and video restoration are utilized to solve the geophysical problem. A spatial point spread function is used to model the resulting effect of wave propagation, migration, and processing. The methodology is compared to current alternatives and discussed in the context of AVO inversion. Good results are obtained in a Barents Sea test case.
\end{abstract}

\section{Introduction}

The concept of superresolution has its origin in seismic inversion. In recent decades, it has found a range of applications in digital image and video restoration. Increased attention to the topic has resulted in theorems defining the limits and in algorithms ready for large-scale problems. Here we show how this approach can be applied in seismic amplitude-variation-with-offset (AVO) inversion and discuss the method in relation to other inversion methods. The methodology we outline gives a type of sparse-spike inversion that is phrased in a spatial context, accounting for the spatial extent of the point spread function as well as the spatial dependencies in a total variation regularization.

Traditionally, AVO methods are categorized as either reflection-based or impedance-based methods. Reflection-based methods tend to work on amplitude maps extracted from peak amplitude, whereas impedance methods work on the full waveform. In the last decade or so, a third line of inversion methods has emerged that can be seen as an augmentation of the impedance methods by including rock-physics models. The superresolution method discussed here is an impedance approach, but it aspires to be a simple inversion that competes with the ease of use of the reflection-based methods.

Comparing the proposed approach to that of using a rockphysics model, it is seen that both methods sharpen the edges by including step changes in the transition. In the facies-based approach, the steps changes are between discrete levels of predefined lithology and fluid classes. The main limitation of the applicability is then the need for these prior classes. The rockphysics approach is thus less suited in regions where rock-physics knowledge is limited. The superresolution approach makes a minimum set of assumptions for the inversion and still manages to coordinate the contrasts in the inversion.

\section{Geophysical roots of superresolution}

The origin of superresolution is in the geophysical tradition. When working with deconvolution, use of the L1 norm rather than the traditional L2 norm as a mean of regularization (Claerbout and Muir, 1973) created a deconvolution consisting of spike trains (Taylor et al., 1979). This approach was thus a sparse-spike inversion. In the noiseless case, the method allowed for perfect reconstruction of the generating spike train (Levy and Fullagar, 1981; Oldenburg et al., 1983). This property was later proved mathematically (Santosa and Symes, 1983, 1986), thus creating the first proof of superresolution. The $1 \mathrm{D}$ case discussed in these papers uses the L1 norm on the reflection coefficients. The impedance relates to the integrated reflection coefficients, so the impedance will have a blocky structure, i.e., step changes at the spike locations. The total variation norm on the impedance corresponds to an L1 norm on the reflection coefficients in the $1 \mathrm{D}$ case. When generalizing the approach to $2 \mathrm{D}$ and $3 \mathrm{D}$, the total variation norm presented in the following provides a natural extension to this $1 \mathrm{D}$ methodology.

\section{Geophysical model}

The geophysical model validity is a primary concern for AVO inversion. In the reflection-based methods, there is an issue due to secondary lobes of the wavelet and tuning effects that are not accounted for in the model. In most impedance and rock-physics approaches, the geophysical model is based on a 1D convolution of a wavelet with the reflection coefficients. This $1 \mathrm{D}$ model does not fully account for the spatial nature of the wave phenomena under study. We formulate the model using a 3D point spread function. In the context of depth-migrated data, an angle stack is related to the elastic impedance, $g_{\theta}$, through a convolution with a point spread function, $\Psi_{\theta}$,

$$
d(x, y, z, \theta)=\Psi_{\theta} * g_{\theta}(x, y, z)+\varepsilon(x, y, z, \theta) .
$$

This geophysical relation is illustrated in Figure 1; see Lecomte et al. (2016) for details. The 3D point spread function is an improvement over the $1 \mathrm{D}$ wavelet, but it does not account for a variable distortion of the overburden as it is assumed to be stationary. The error term $\varepsilon$ is included to highlight that the relation is an approximation. In a regular grid, we get

$$
d_{\theta}=\Psi_{\theta} g_{\theta}+\varepsilon_{\theta}
$$

where the operator $\Psi_{\theta}$ is diagonalized by the discrete 3D Fourier transform; thus, it is efficiently stored and computed. The parameter

${ }^{1}$ Lundin-Norway, Lundin Geolab, Lysaker, Norway. E-mail: odd.kolbjornsen@lundin-norway.no; andreas-kjelsrud.evensen@lundin-norway.no; espen-harris.nilsen@lundin-norway.no; jan-erik.lie@lundin-norway.no.

${ }^{2}$ University of Oslo, Department of Mathematics, Oslo, Norway. 

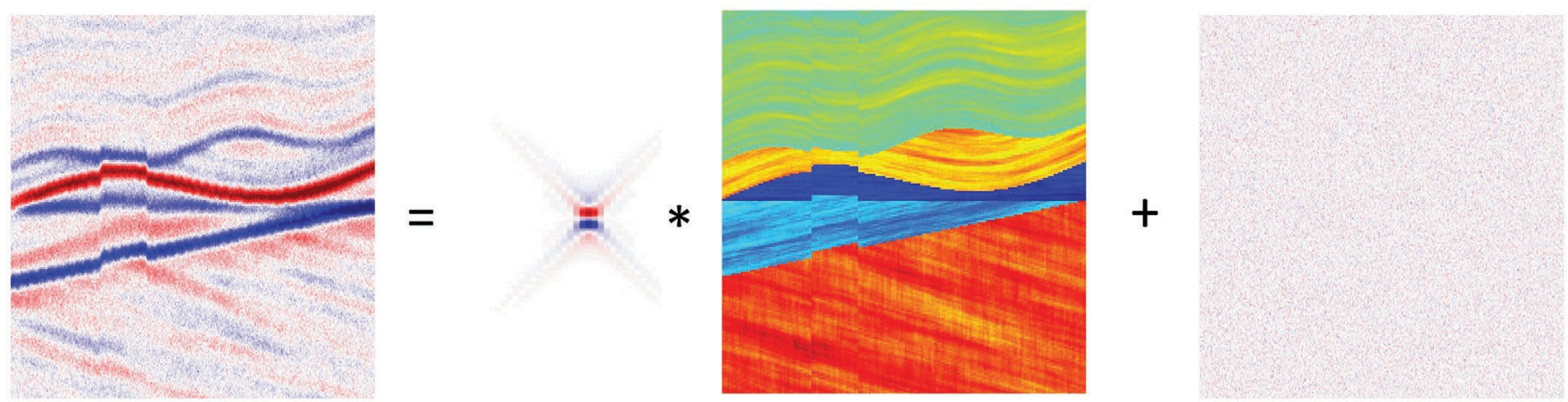

Figure 1. Spatial convolution. Data is to the left. In the middle left is the point spread function, and to the middle right is the geophysical parameter. The noise is to the far right.

of the geophysical relation is linked to a selected set of earth parameters $m$ through a linear relation.

$$
g_{\theta_{l}}=A_{l} m
$$

The matrix $\boldsymbol{A}_{\boldsymbol{l}}$ typically corresponds to the linearized Zoeppritz equation; thus, it is represented with three numbers in each spatial location. The earth parameters are the targets of the inversion.

\section{Regularization}

The lack of resolution in the point spread function $\Psi_{\theta}$ and the weak third-order effect in the AVO relations $A_{l}$ makes the inverse problem ill posed. In the theory of superresolution, the total variation norm provides one option. The total variation norm promotes solutions that have constant values within unspecified regions. This is a perfect match for an earth model consisting of blocks with a layered structure. For a multiparameter inversion, there are three basic forms for the total variation:

$$
\begin{gathered}
\|\boldsymbol{m}\|_{A T V}=\sum_{i=1}^{N} \sum_{j=1}^{p} \beta_{j}\left(\left|\nabla_{x} m_{j, i}\right|+\left|\nabla_{y} m_{j, i}\right|+\left|\nabla_{z} m_{j, i}\right|\right), \\
\|\boldsymbol{m}\|_{T V}=\sum_{i=1}^{N} \sum_{j=1}^{p} \beta_{j} \sqrt{\left|\nabla_{x} m_{j, i}\right|^{2}+\left|\nabla_{y} m_{j, i}\right|^{2}+\left|\nabla_{z} m_{j, i}\right|^{2}}, \\
\|\boldsymbol{m}\|_{M T V}=\sum_{i=1}^{N} \sqrt{\sum_{j=1}^{p} \beta_{j}^{2}\left(\left|\nabla_{x} m_{j, i}\right|^{2}+\left|\nabla_{y} m_{j, i}\right|^{2}+\left|\nabla_{z} m_{j, i}\right|^{2}\right)} .
\end{gathered}
$$

These norms can be compared to the corresponding weighted L2 norm:

$$
\|\boldsymbol{m}\|_{L 2}=\sqrt{\sum_{i=1}^{N} \sum_{j=1}^{p} \beta_{j}^{2}\left(\left|\nabla_{x} m_{j, i}\right|^{2}+\left|\nabla_{y} m_{j, i}\right|^{2}+\left|\nabla_{z} m_{j, i}\right|^{2}\right)}
$$

The outer sum is over all elements in a grid, the inner sum is over the parameters, and the summands are the gradients of the earth parameters. The weights $\left(\beta_{i}, j=1, \ldots, p\right)$ are proportional to one over the standard deviation of the corresponding earth parameter and make the problem independent of scale. The key feature of the total variation norm is that it promotes solutions having many zeros in the gradients. In the iterations, we observe that terms within a square root are evaluated together in a thresholding step. Thus, terms within the same square root will synchronize the zeros. This has the following implications. The anisotropic total variation norm promotes solutions with griddependent discontinuities, as it sets the threshold for each individual term of the gradient independently. The second norm denotes that the isotropic total variation norm is grid independent and promotes the gradients of each individual earth parameter to be zero independently. Thus, it creates contrasts in the individual earth parameters, but the strong contrasts in two earth parameters do not have a preference for colocation. The multivariate isotropic total variation norm evaluates the weighted norm of all gradients simultaneously and thereby promotes simultaneous nonzero gradients; thus, it leans toward colocated contrasts. The weighted L2 norm (or the square of it) does not promote zeros, as all elements are within the square root. Thus, we argue that the desired regularization is the multivariate isotropic total variation norm as this will favor synchronization contrasts and have gridindependent solutions. The problem is then given as

$$
\begin{aligned}
& \text { Minimize }: \sum_{l=1}^{n_{L}} \frac{\mu_{l}}{2}|| \boldsymbol{d}-\left.\Psi_{\theta_{l}} \boldsymbol{g}_{\theta_{l}}\right|^{2}+\|\boldsymbol{m}\|_{M T V} \\
& \text { Subject to : } \boldsymbol{A}_{\boldsymbol{l}} \boldsymbol{m}=\boldsymbol{g}_{\theta_{l}}, \quad l=1, \ldots, n_{L},
\end{aligned}
$$

where the additional parameters $\mu_{l}, l=1, \ldots, n_{L}$ provide the trade-off between data and regularization.

\section{Solving the problem}

The problem is a standard convex optimization problem. There are currently numerous methods for solving these. In our implementation, we have used a scheme with relaxation and iterations with an alternating direction method (ADM) (see Chan et al., 2011). The key feature of this approach is that the total variation norm is replaced with a slack variable related to the gradient of the earth parameter. This splits the problem into two smaller problems in each iteration. The first problem is the spatially coupled geophysical inverse problem that is solved in the Fourier domain using an algorithm with complexity $N \log N$. The second problem is related to the nonlinear part (i.e., the total variation norm), and it is solved for each grid location 
independently. During iterations, the slack is gradually decreased to obtain convergence. For further details on the ADM in the context of seismic data, see Kolbjørnsen and Evensen (2019); see also Wang et al. (2018) for use of total variation in seismic inversion. There is no additional computational cost for including the spatial point spread function as compared to the $1 \mathrm{D}$ wavelet since the computations are done in Fourier domain. It is also straightforward to introduce colored noise, thus enabling filtering methods such as automatic factorial cokriging within the framework. The standard weighted L2 solution to the inversion problem is solved with computations corresponding to one iteration. To complete the ADM algorithm, the number of iterations needed is most often on the order of 10 to 100 . Thus there is an additional complexity compared to the weighted L2. The total computation is well within reach of a standard CPU.

\section{The background model}

The background model is always an issue in AVO methods. In the reflection-based models, this entails constraining the properties of the layer above or below the target zone. For the impedance methods, this corresponds to inclusion of a lowfrequency background model. Traditionally the background model is obtained by interpolating wells guided by horizons interpreted from the seismic. One of the benefits of introducing rock-physics models is that it formalizes a framework for estimation of multiple rock-physics trends, one for each lithology class. This apparently resolves the issue of the background model, but trends still need to be estimated relative to some predefined quantity in order to extrapolate away from wells. Failure to identify the right parameterization will ultimately result in erroneous conclusions. An alternative is to abandon the background model and consider a relative inversion. This approach struggles with mean reversion in regions of constant impedance, and the common L2 regularization tends to smooth out edges of a blocky earth. The benefit of the total variation norm is that it encourages few large leaps and discourages small adjustments, so the approach tends to favor abrupt changes and mitigate mean reversion. Thus, the approach is well suited for relative inversion. The form of the inverse problem outlined earlier is more general than that of AVO data, so it is possible to include a low-frequency model by including an additional data set. In the "background data set" the data is the low-frequency model, and the point spread function is the smoothing kernel, which gives the low-frequency model.

\section{Synthetic example}

As a synthetic example, consider the earth parameters to be $\log$ transform of acoustic impedance, velocity ratio, and density, and assume that we have partial stacks of $5^{\circ}, 15^{\circ}, 25^{\circ}$, and $35^{\circ}$ in addition to the background model. Figure 2 shows the model setup with the ground truth and the background model. Figure 3 shows the point spread function of the seismic data together with the smoothing kernel of the background model. The mapping from earth parameters to the geophysical parameters are

$$
\boldsymbol{A}^{T}=\left(\begin{array}{ccccccc}
0.496 & 0.469 & 0.430 & 0.416 & 1.0 & 0.0 & 0.0 \\
0.008 & 0.067 & 0.179 & 0.329 & 0.0 & 1.0 & 0.0 \\
0.000 & -0.002 & -0.019 & -0.081 & 0.0 & 0.0 & 1.0
\end{array}\right) \text {. }
$$

The first terms are from the linearized Zoeppritz equation for earth. The identity matrix at the end is from the background model. Figure 4 shows the seismic data with white noise added. Inversion is performed for the total variation norm and the weighted L2 norm. In both inversions, the data weight is set as the optimal value. The inversion results are displayed in Figure 5,
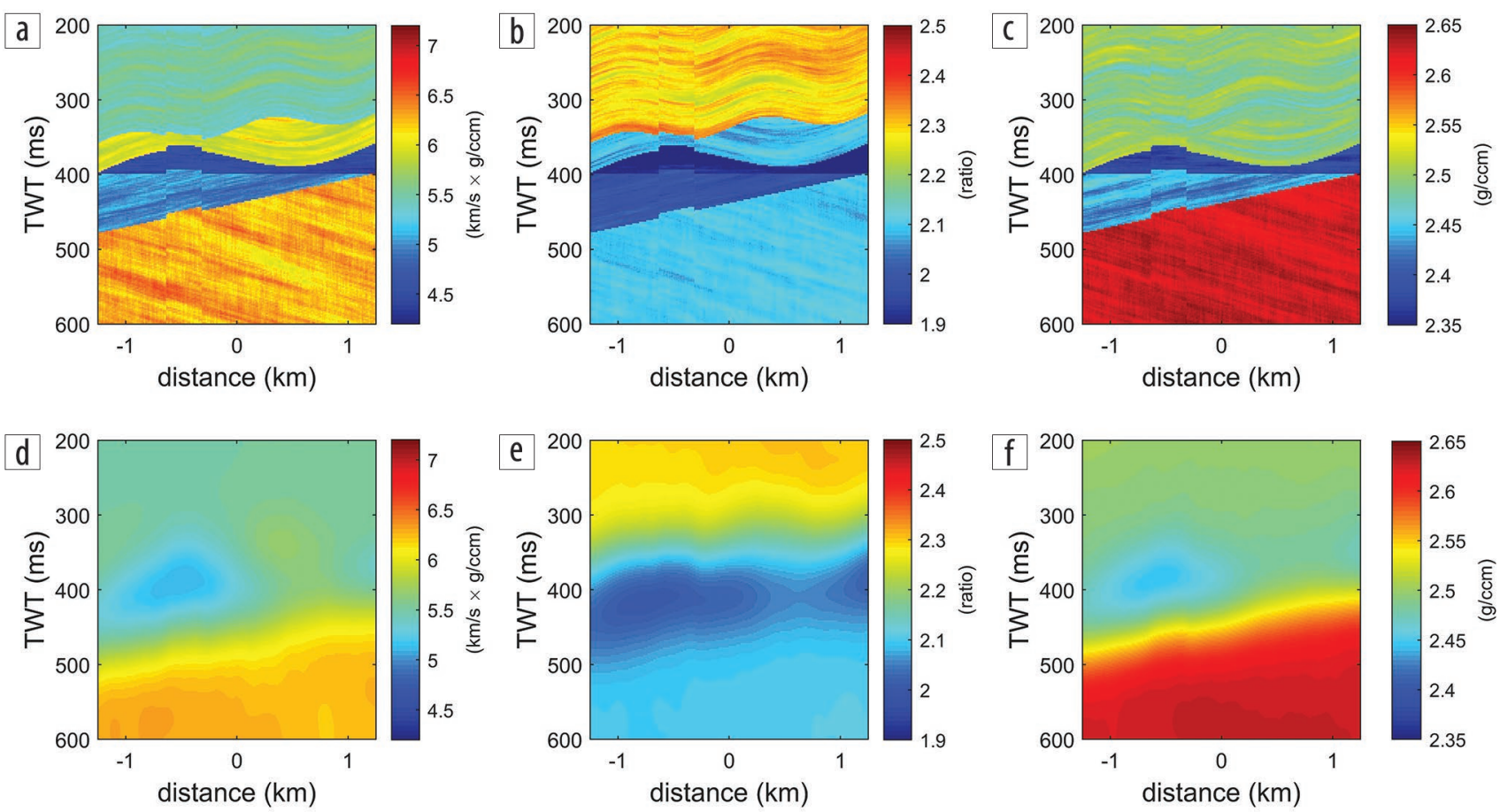

Figure 2. Model and background. (a-c) Ground truth. (d-f) Background model. (a) and (d) Acoustic impedance. (b) and (e) Velocity ratio. (c) and (f) Density. 

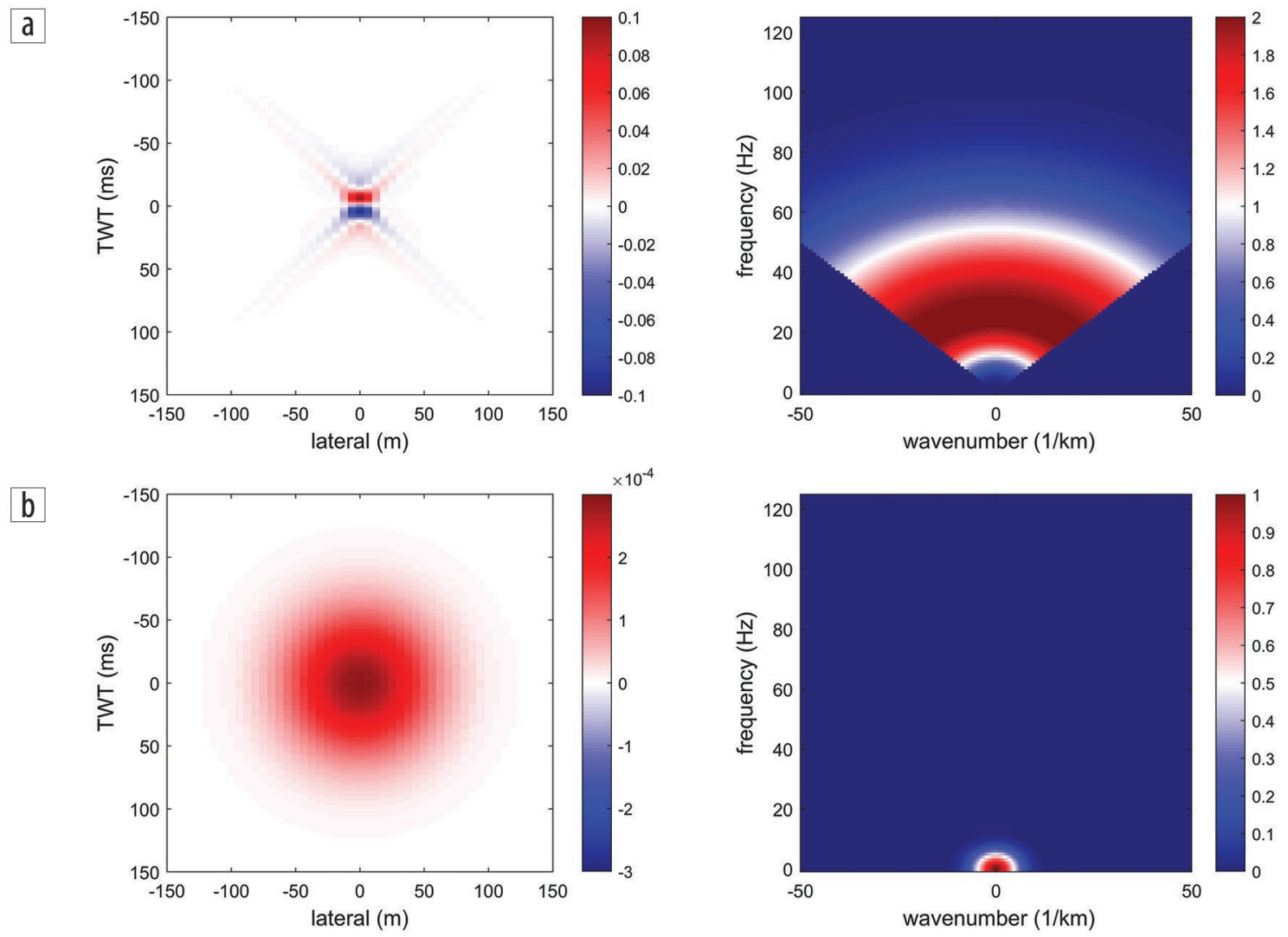

Figure 3. Point spread function and spectrum. (a) The point spread function and the magnitude of the corresponding spectrum in $f-k$ domain. (b) The same plot for the kernel defining the background model.
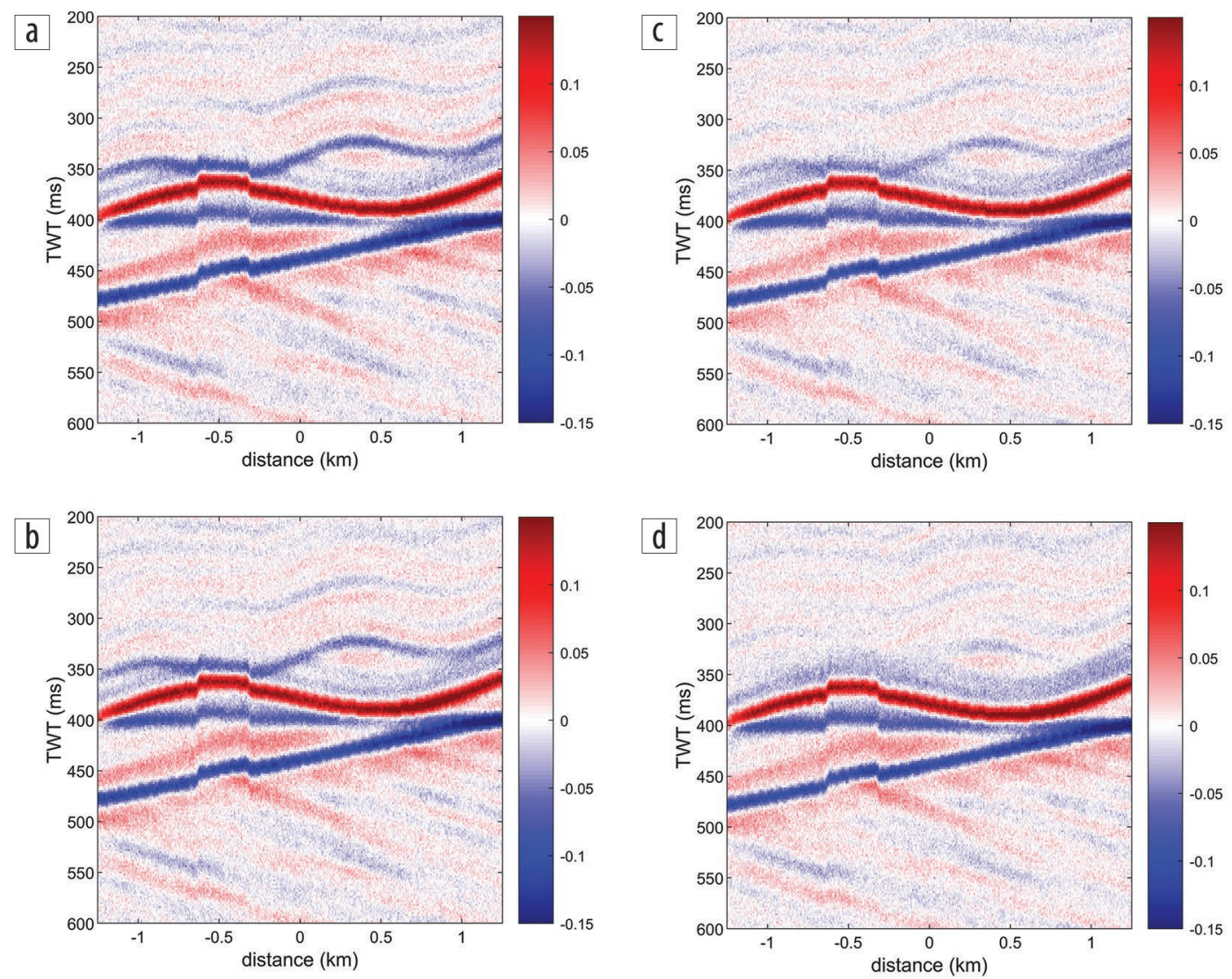

Figure 4. Data with noise. (a) $5^{\circ}$ stack. (b) $15^{\circ}$ stack. (c) $25^{\circ}$ stack. (d) $35^{\circ}$ stack. 
and Figure 6 shows the deviation from the ground truth. The root mean square of this deviation is summarized in Table 1 . The total variation norm improves the results of weighted L2 inversion by more than 30 percent. For the acoustic impedance, the edges are sharpened. For the velocity ratio, the level is kept to a larger extent when moving away from the contrasts, and even for the density, some structure is seen. The results for the three parameters illustrate typical behavior of the two inversion schemes.

To compare the results with standard AVO analysis, we have compared the reflection coefficients of the three earth parameters to that of the data and ground truth in Figure 7. The seismic data sets are obtained by inverting the pointwise relation of the data
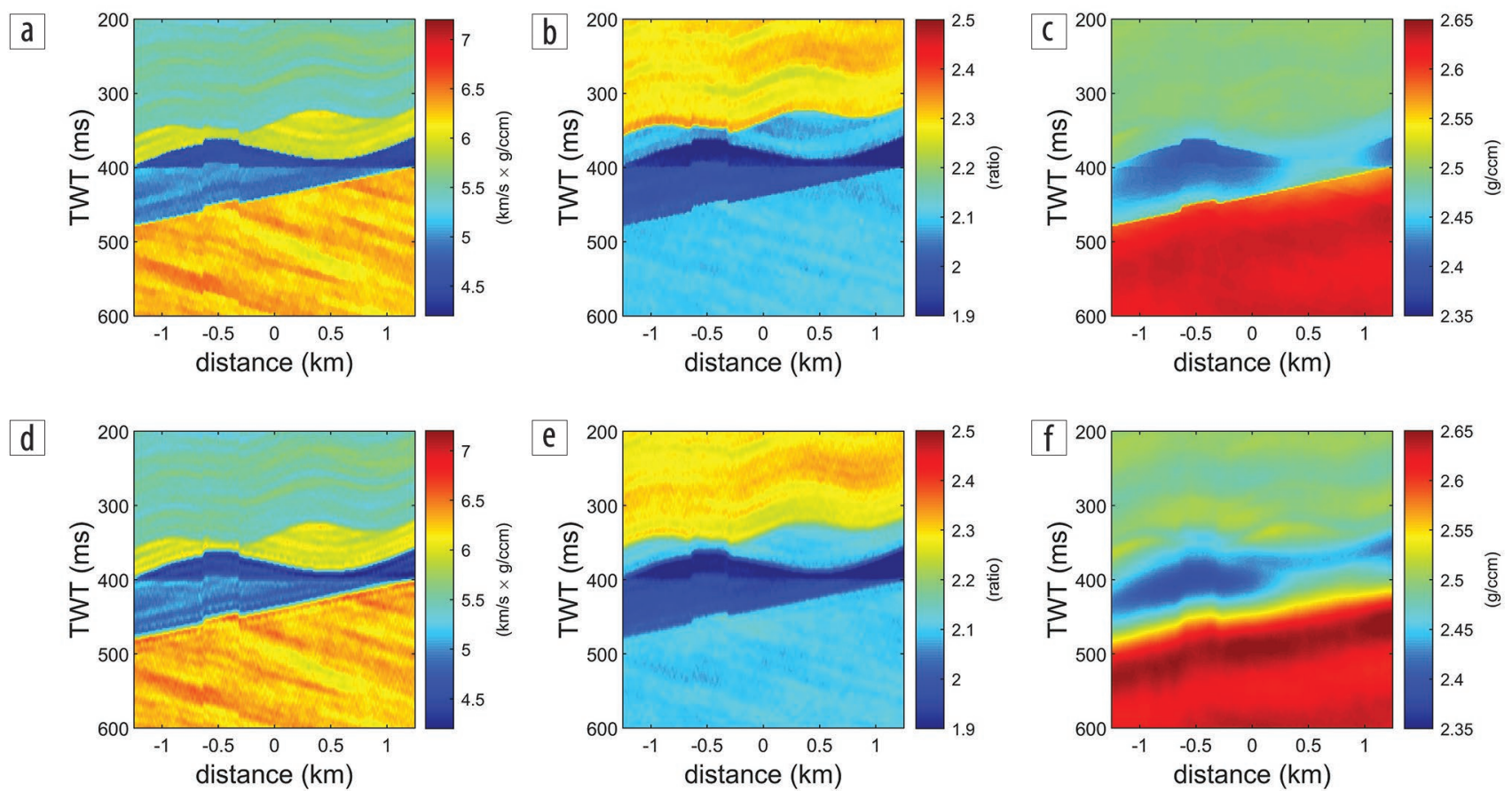

Figure 5. Inversion result. Inversion for (a-c) total variation and (d-f) weighted L2 norm. (a) and (d) Acoustic impedance. (b) and (e) Velocity ratio. (c) and (f) Density.
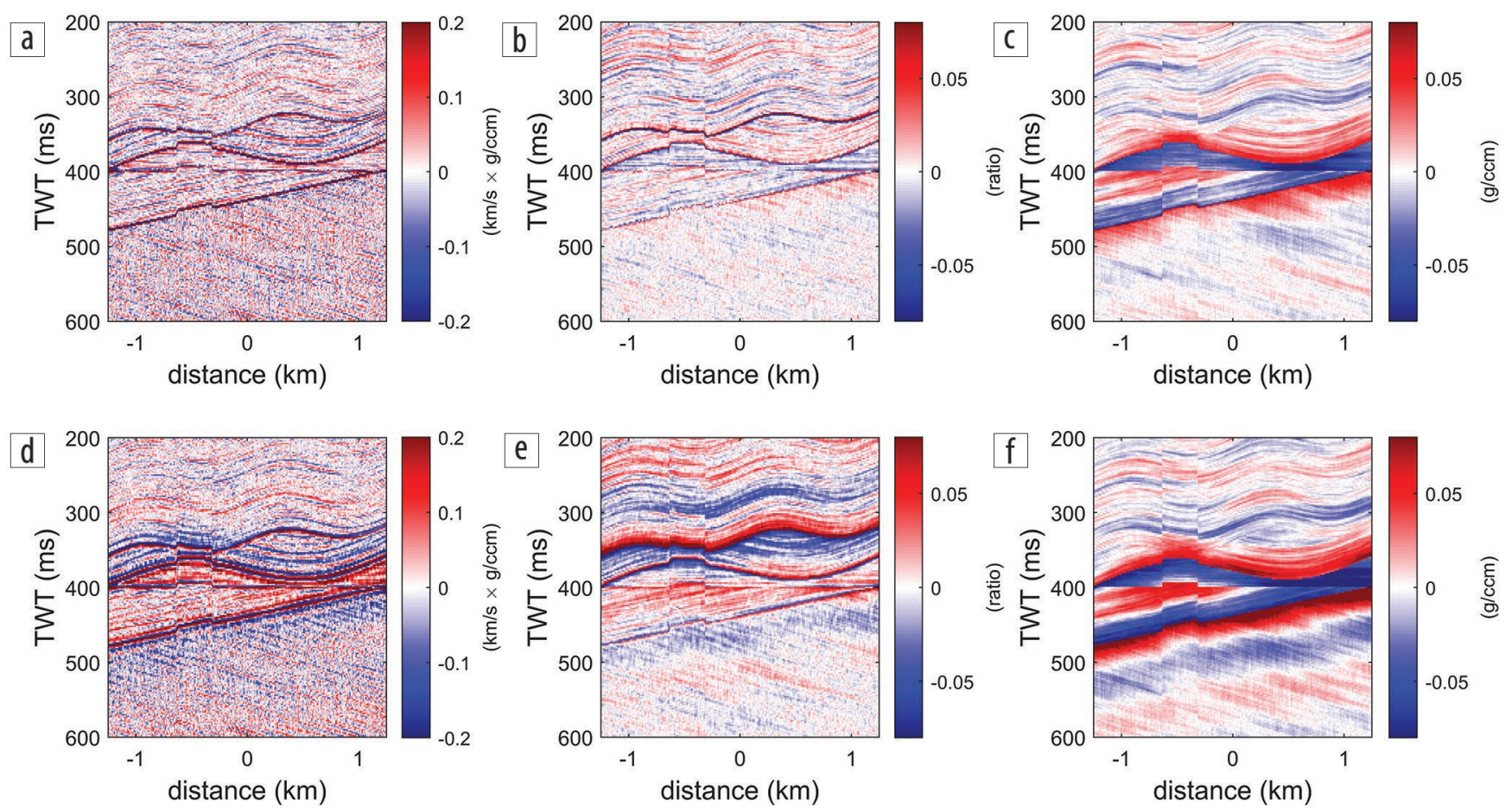

Figure 6. Inversion difference to ground truth. Difference for (a-c) total variation and (d-f) weighted L2. (a) and (d) Acoustic impedance. (b) and (e) Velocity ratio. (c) and (f) Density. 
directly. Two different methods are used, a damped inversion $A_{D}^{\dagger}$ and a pseudo inverse $A^{\dagger}$ :

$$
A_{D}^{\dagger}=\left(\begin{array}{cccc}
0.723 & 0.610 & 0.414 & 0.205 \\
-0.575 & -0.282 & 0.256 & 0.924 \\
0.028 & 0.032 & 0.013 & -0.090
\end{array}\right)
$$

Table 1. Deviation from ground truth. Root mean square from the two inversions. Deviation from background model is included for reference.

\begin{tabular}{lc|c|c|} 
& \multicolumn{1}{c}{$\begin{array}{c}\text { Acoustic } \\
\text { impedance }\end{array}$} & \multicolumn{1}{c}{$\begin{array}{c}\text { Velocity } \\
\text { ratio }\end{array}$} & Density \\
Background & 0.0636 & 0.0261 & 0.0155 \\
WL2 inversion & 0.0195 & 0.0115 & 0.0131 \\
\hline MATV inversion & 0.0139 & 0.0073 & 0.0088 \\
\hline
\end{tabular}

a

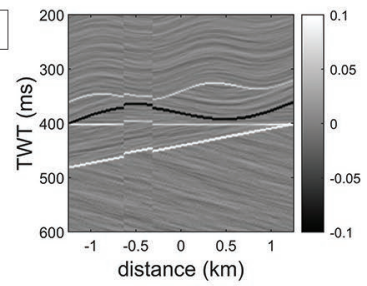

b

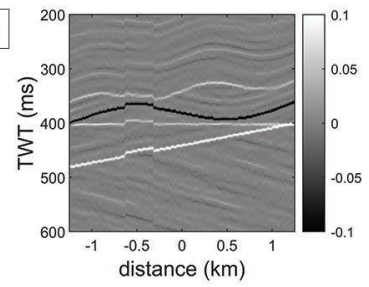

C

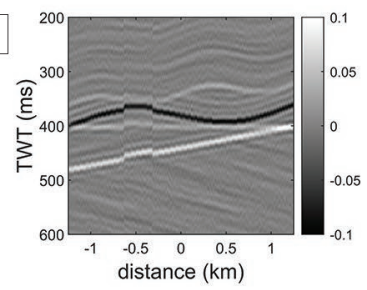

d

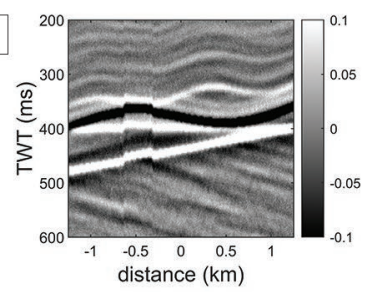

e

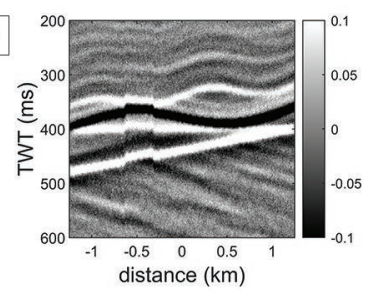

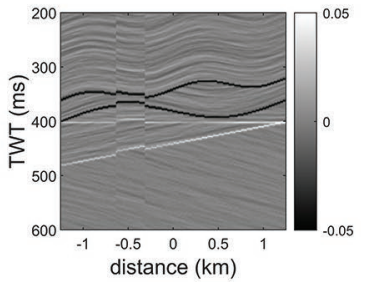
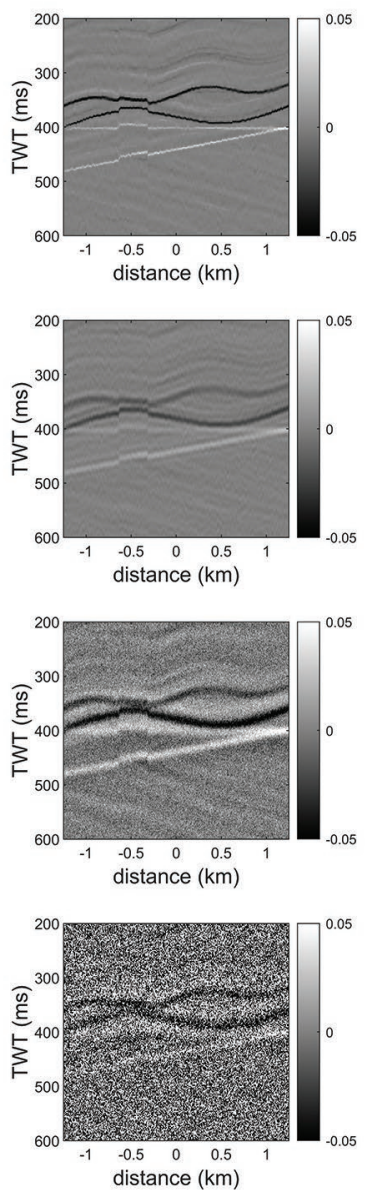

$$
A^{\dagger}=\left(\begin{array}{cccc}
1.737 & 0.689 & -0.535 & 0.109 \\
-7.5916 & 1.135 & 9.761 & -2.318 \\
22.348 & 8.8918 & 36.488 & -21.125
\end{array}\right) \text {. }
$$

In Figure 7, it is seen that both choices work to some extent for the acoustic impedance and velocity ratio. For the density, however, there is no information left. The pseudo inverse $A^{\dagger}$ creates an image containing only noise, and the damped inversion $A_{D}^{\dagger}$ creates an image in conflict with the original.

\section{Superresolution}

Current theoretical results of superresolution for total variation norms allow for resolving all frequencies in a signal even with a limited bandwidth in the observations. (Candès and Fernandez-Granda, 2014). However, this promise is conditioned to events being sufficiently well separated in the original signal.

If the separation of geologic events

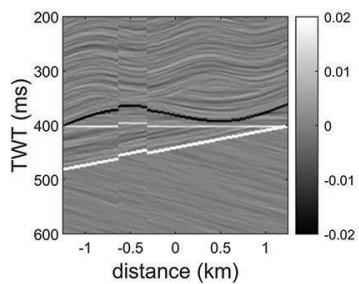
approaches the Rayleigh limit, it is observed that odd events have a repulsion and cancel the magnitudes of the events, and even events tend to merge. This gives a simple understanding of how the methodology will influence the inversion results. Large-scale features will be well resolved including all frequencies of these events. In layers with a step change compared with the background, we find that thin layers with large contrast will tend to reproduce thicker layers with weaker contrasts where the contrast is such that the elastic impedance thickness matches that of the original. In the case where the thin layer is a part of a stair-step trend, the layer will tend to be thinner or will disappear, but the level of the combined step will be largely resolved. The total variation norm is originally used for noise removal (see, e.g., Rudin et al., 1992; Strong and Chan, 2003). This formulation is also considered in the geophysical context (Gholami, 2015). It is known that in the setting of noise removal, the total variation approach tends to reproduce edge locations but underestimates the magnitude of the contrast. This issue is commonly denoted "contrast loss." In the synthetic example, the contrast loss is perhaps best illustrated in layer 3 of the velocity ratio in Figure 6. The deviation from ground truth tends to be light blue inside the entire zone due to contrast loss. In comparison, the L2 approach goes from dark blue on the top to light red on the bottom. The

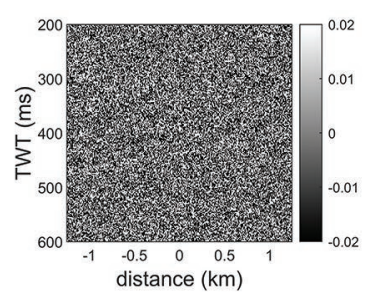

Figure 7. Earth parameter contrasts. The left column shows contrasts in acoustic impedance. The middle column shows contrasts in velocity ratio. The right column shows contrasts in density. (a) Ground truth. (b-e) Estimated contrasts. (b) MTV inversion. (c) Weighted L2 inversion. (d) Damped point inversion, i.e., multiplying data cubes with $A_{D}^{\dagger}$. (e) Point inversion without damping, i.e., multiplying data set with $A^{\dagger}$. 
total variation norm is well studied; Chan et al. (2006) list a set of caveats in use.

There are also theoretical results for any methodology of superresolution that state that any method that is able to resolve events with a large overlap - i.e., give different interpretations to two very similar data sets - will be unstable (N. Gottschling et al., personal communication, 2019). This will be the case for any type of inversion regardless of the origin, whether neural nets (Antun et al., 2019) or regularization with sophisticated norms. This is also the main argument for using a probabilistic approach to the inversion. In the probabilistic approach, one considers the possibility of multiple solutions to the problem, all of which contain the full frequency span. The probabilistic approach is used in most rockphysics methods, but in some applications, the prior is used just for regularization. In these cases, the method might be unstable. As an example, take the case of a discrete algorithm: a small change in seismic amplitudes can change the odds in favor a different mode as the global optimizer. The main advantage with the superresolution approach based on total variation is that it comes with a guarantee of stability and that the error modes of the methodology are well established.

\section{Barents Sea case}

We have applied the methodology on a data set acquired in the Barents Sea. It is possible to estimate the point spread function by combining illumination vectors from ray tracing and well data (Georgsen et al., 2010). In our application, we have used a simpler data-driven approach by setting

$$
\Psi(x, y, t)=c \cdot T(x, y, t) \mathcal{F}_{3 D}^{-1}\left(-i \omega\left|\hat{d}\left(k_{x}, k_{y}, \omega\right)\right|\right),
$$

where $\hat{d}$ is the $3 \mathrm{D}$ Fourier transform of the seismic data, $\mathcal{F}_{3 D}^{-1}$ is the inverse Fourier transform, $T$ is a tapering function having a lateral kernel width comparable to the Fresnel zone, and $c$ is an amplitude scale. We use the full stack for estimation of a common point spread function. In our limited experience, we have found that this provides reasonable results in the early phase of exploration of true amplitude processed data. The data survey has a source above streamer configuration giving both positive and negative offset (Dhelie et al., 2018). The data went through a noise attenuation/conditioning flow prior to inversion. Negative and positive offsets are processed separately providing two near stacks and two far stacks. The same data set has been utilized previously for enhancing the full stack using total variation with good results. Thus, rather than including the mid-stack, we utilize the full stack. Figure 8 shows the full stack and the average far minus near stack. Rather than inverting for the elastic parameters, we invert for full-stack elastic impedance and gradient impedance (far-near). Input cubes are the full stack and the four alternative computations of far-near. Thus, the matrix relating data to inversion parameters is

$$
A=\left(\begin{array}{ccccc}
1.0 & 0.0 & 0.0 & 0.0 & 0.0 \\
0.0 & 1.0 & 1.0 & 1.0 & 1.0
\end{array}\right)
$$

In this case, the geophysical problem separates, but the multivariate isotropic total variation norm couples the problems such that they need to be solved simultaneously. Figure 9 shows the inversion result. The absolute scale is unknown due to the lack of scaling of the point spread function. The correlation between the two inverted properties is moderate $(-0.5)$, indicating that the offset stack is not dominated by the acoustic impedance. A particularly interesting feature is the structure at about $6.2 \mathrm{~km}$, $200 \mathrm{~ms}$. In the full stack-impedance (Figure 9a), it is clearly seen as a soft (white) structure below an apparent closure. The gradient impedance (Figure 9b) also shows a low (white region as well),
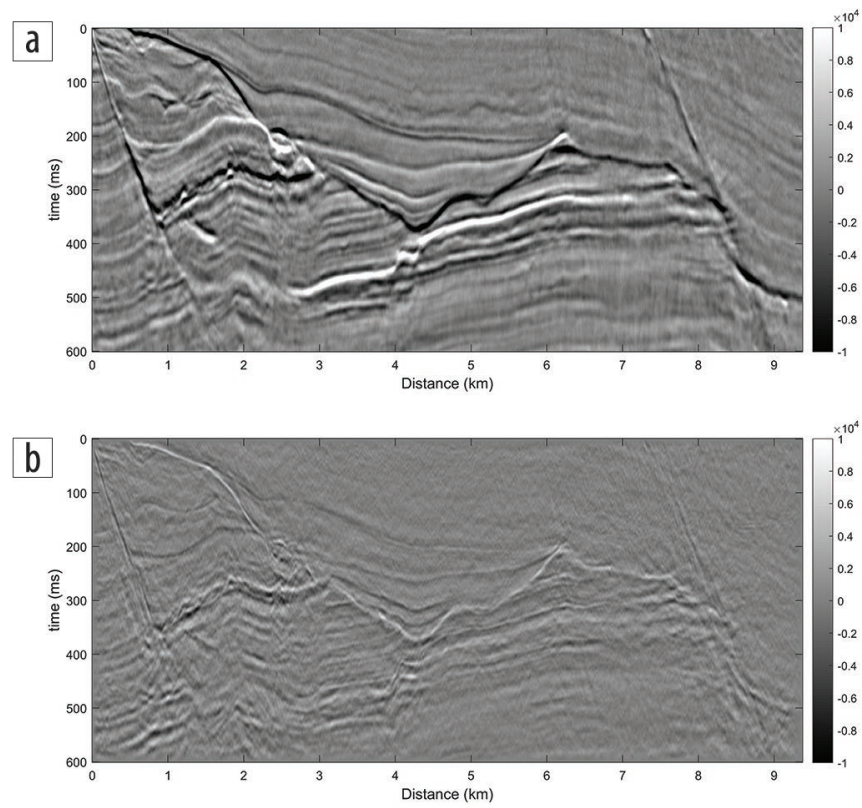

Figure 8. Barents Sea data. (a) Full stack. (b) Far minus near stack. Tapering is applied along the edges.
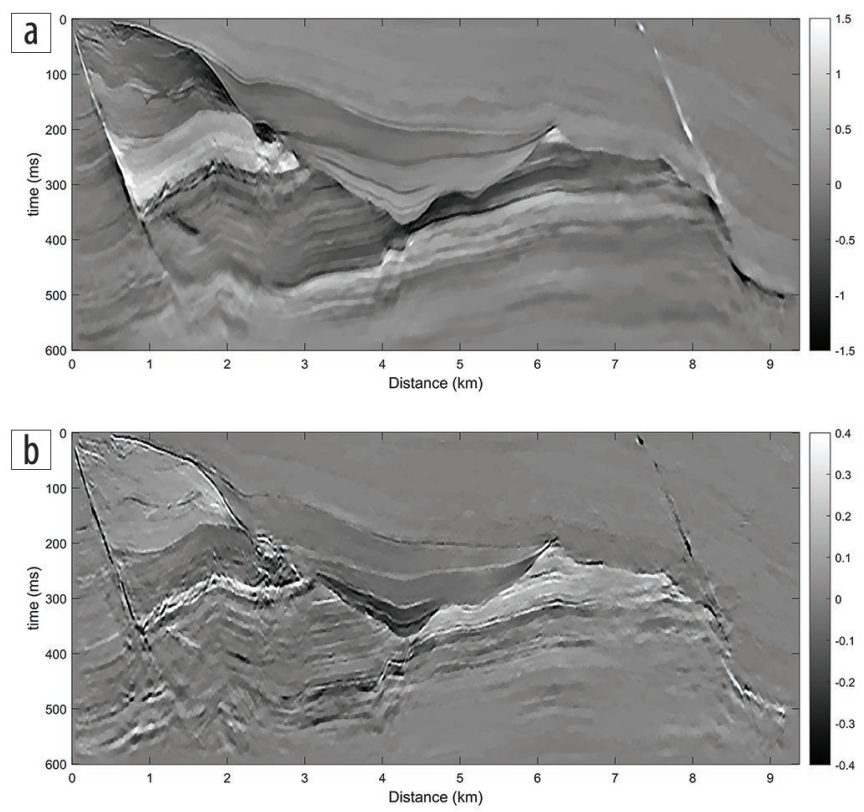

Figure 9. Barents Sea inversion: (a) Inverted full-stack impedance. (b) Inverted gradient impedance (far-near). 
but this region extends much farther down. This is a typical indication of a partially fluid-filled sand. A well through the structure has shown that the segment contains gas. The tuning parameters $(\mu, l=1, \ldots, 5)$ were selected by trial and error. The residual energy is $5 \%$ for the full stack and $40 \%$ for each of the offset stacks. The energy in the intercept cubes is about $20 \%$ of that in the full stack, so the energy in the residuals from the full stack and the energy in the average residual of the four intercept stacks are of the same magnitude. The most dominant feature in the residuals are in regions around the faults. This might be due to imperfections in the spatial point spread function. An interesting task for the future is to improve the estimates of the spatial point spread function and use physics-based models as is done in leastsquares migration (Fletcher et al., 2016).

\section{Use of prior information}

The inversion we outlined previously is designed to require minimal input to the inversion, and we currently use it for interpreting relative features such as softer and harder. This is meaningful in a screening process in which the target is to increase the regional understanding, stimulate new geologic ideas, and generate new plays. It is possible, however, to push the approach further by imposing additional prior information in the inversion. The issue of a background trend is discussed earlier; another option is to use spatially variable coefficients in the total variation norm:

$$
\|\boldsymbol{m}\|_{M T V}=\sum_{i=1}^{N} \sqrt{\sum_{j=1}^{p} \beta_{i, j}^{2}\left(\left|\nabla_{x} m_{j, i}\right|^{2}+\left|\nabla_{y} m_{j, i}\right|^{2}+\left|\nabla_{z} m_{j, i}\right|^{2}\right)} .
$$

To be specific, we want to model rock hardening with depth and that there is a collection of alternative lithology fluid classes
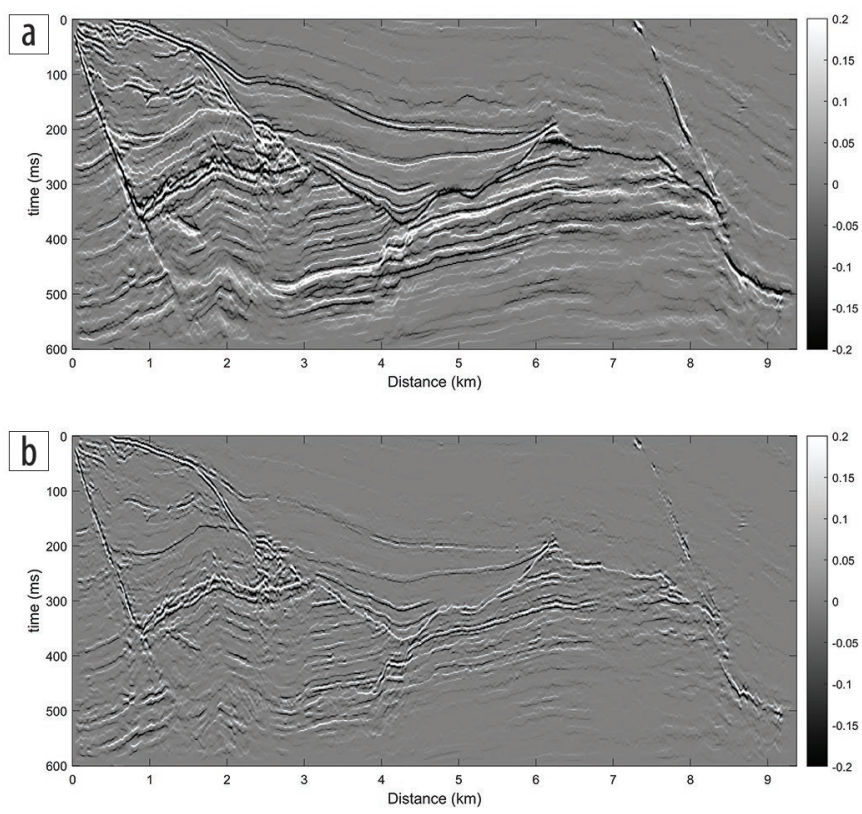

Figure 10. Barents Sea reflectivity inversion. (a) Inverted full-stack reflectivity. (b) Inverted gradient reflectivity (far-near). displaying different trends. The average trend of all lithology fluid classes is modeled using a background trend. The selection of spatially variable coefficients $\left(\beta_{i, j}^{2}\right)$ depends on whether the trends for different lithology fluid classes converge or diverge with depth. If the contrasts between trends decrease with depth, the coefficients $\left(\beta_{i, j}^{2}\right)$ should increase. Decreasing coefficients models diverging trends. Each earth parameter is evaluated independently.

\section{Closing remark}

The theory of superresolution using sparsity measures is well established, and we have shown that the method can be utilized in the setting of AVO inversion. The result is an inversion method that requires minimal input and thus avoids detailed prior choices that might bias the result. In this way, the method compares to that of reflection-based methods, and we argue that it is well suited to the exploration setting where certain prior information can be sparse. We have established a workflow that enables many options for further development, the main improvement being the estimation of the point spread function. We currently use a statistical estimation procedure, but a more sophisticated approach could be based on geophysical relations, imaging operators, and well data. As for all AVO inversion, a hands-on relationship to the quality of the angle stacks used is important. Ideally the workflow should be integrated with the data processing, with full control over the velocity fields and mutes applied. ILE

\section{Data and materials availability}

Data associated with this research are confidential and cannot be released.

Corresponding author: odd.kolbjornsen@lundin-norway.no

\section{References}

Antun, V., F. Renna, C. Poon, B. Adcock, and A. C. Hansen, 2019, On instabilities of deep learning in image reconstruction - Does AI come at a cost?: arXiv:1902.05300v1.

Candès, E. J., and C. Fernandez-Granda, 2014, Towards a mathematical theory of super-resolution: Communications on Pure and Applied Mathematics, 67, no. 6, 906-956, https://doi.org/10.1002/ cpa. 21455 .

Chan, T., S. Esedoglu, F. Park, and A. Yip, 2006, Total variation image restoration: Overview and recent developments, in $\mathrm{N}$. Paragios, Y. Chen, and O. Faugeras, eds., Handbook of mathematical models in computer vision: Springer, 17-31, https://doi. org/10.1007/0-387-28831-7_2.

Chan, S. H., R. Khoshabeh, K. B. Gibson, P. E. Gill, and T. Q. Nguyen, 2011, An augmented Lagrangian method for total variation video restoration: IEEE Transactions on Image Processing, 20, no. 11, 3097-3111, https://doi.org/10.1109/ TIP.2011.2158229.

Claerbout, J. F., and F. Muir, 1973, Robust modeling with erratic data: Geophysics, 38, no. 5, 826-844, https://doi.org/10.1190/1.1440378.

Dhelie, P. E., V. Danielsen, J. E. Lie, A. K. Evensen, A. Wright, N. Salaun, J.-L. Rivault, et al., 2018, Improving seismic imaging in the Barents Sea by source-over-cable acquisition: $88^{\text {th }}$ Annual International Meeting, SEG, Expanded Abstracts, 71-75, https:// doi.org/10.1190/segam2018-2998198.1. 
Fletcher, R. P., D. Nichols, R. Bloor, and R. T. Coates, 2016, Leastsquares migration - Data domain versus image domain using point spread functions: The Leading Edge, 35, no. 2, 157-162, https://doi.org/10.1190/tle35020157.1.

Georgsen, F., O. Kolbjørnsen, and I. Lecomte, 2010, A 3d ray-based pulse estimation for seismic inversion of PSDM data: $72^{\text {nd }}$ Conference and Exhibition, EAGE, Extended Abstracts, https:// doi.org/10.3997/2214-4609.201400607

Gholami, A., 2015, Nonlinear multichannel impedance inversion by total-variation regularization: Geophysics, 80, no. 5, R217R224, https://doi.org/10.1190/geo2015-0004.1.

Kolbjørnsen, O., and A. K. Evensen, 2019, Digital superresolution in sesmic amplitude processing: $89^{\text {th }}$ Annual International Meeting, SEG, Expanded Abstracts, 579-584, https:/doi. org/10.1190/segam2019-3214317.1.

Lecomte, I., P. L. Lavadera, C. Botter, I. Anell, S. J. Buckley, C. H. Eide, A. Grippa, V. Mascolo, and S. Kjoberg, 2016, 2(3)D convolution modelling of complex geological targets beyond 1D convolution: First Break, 34, no. 5, 99-107.

Levy, S., and P. K. Fullagar, 1981, Reconstruction of a sparse spike train from a portion of its spectrum and application to highresolution deconvolution: Geophysics, 46, no. 9, 1235-1243, https://doi.org/10.1190/1.1441261.

Oldenburg, D. W., T. Scheuer, and S. Levy, 1983, Recovery of the acoustic impedance from reflection seismograms:
Geophysics, 48, no. 10, 1318-1337, https://doi.org/10.1190/1. 1441413.

Rudin, L. I., S. Osher, and E. Fatemi, 1992, Nonlinear total variation based noise removal algorithms: Physica D. Nonlinear Phenomena, 60, no. 1-4, 259-268, https://doi.org/10.1016/01672789(92)90242-F.

Santosa, F., and W. W. Symes, 1983, Inversion of impedance profile from band-limited data: Digest - International Geoscience and Remote Sensing Symposium (IGARSS), IEEE.

Santosa, F., and W. W. Symes, 1986, Linear Inversion of BandLimited Reflection Seismograms: SIAM Journal on Scientific and Statistical Computing, 7, no. 4, 1307-1330, https://doi. org/10.1137/0907087.

Strong, D., and T. Chan, 2003, Edge-preserving and scale-dependent properties of total variation regularization: Inverse Problems, 19, no. 6, S165-S187, https://doi.org/10.1088/0266-5611/ 19/6/059.

Taylor, H. L., S. C. Banks, and J. F. McCoy, 1979, Deconvolution with the L1 norm: Geophysics, 44, no. 1, 39-52, https://doi. org/10.1190/1.1440921.

Wang, D., J. Gao, and H. Zhou, 2018, Data-driven multichannel seismic impedance inversion with anisotropic total variation regularization: Journal of Inverse and Ill-Posed Problems, 26, no. 2, 229-241, https://doi.org/10.1515/jiip-2017-0024. 